\title{
\& Research Square

\section{Three-dimensional Real-time Triplane versus Biplane Simpson for left ventricular ejection fraction estimation after ST-Segment Elevation Myocardial Infarction}

Diogo Rodrigues Brás ( $\sim$ dbras@hevora.min-saude.pt )

Hospital Espirito Santo Evora https://orcid.org/0000-0003-3327-5054

Carla Reis

Hospital Santa Cruz

Eduarda Horta

Hospital Santa Cruz

Miguel Mendes

Hospital Santa Cruz

Maria João Andrade

Hospital Santa Cruz

\section{Research Article}

Keywords: 3D Echocardiography, Real-time triplane, LV ejection fraction, ST-segment elevation myocardial infarction

Posted Date: March 30th, 2021

DOI: https://doi.org/10.21203/rs.3.rs-163572/v1

License: (c) (i) This work is licensed under a Creative Commons Attribution 4.0 International License.

Read Full License 


\section{Abstract}

Purpose: The Biplane Simpson (BPS) remains the recommended 2-dimensional method to assess left ventricular ejection fraction (LVEF). However, it has known limitations that may be overcome with 3D realtime triplane (RTTP), for instance, obtaining all apical views simultaneously at the same cardiac cycle. Accurate LVEF measurement is of utmost importance, as it guides the allocation of many treatments that reduce morbidity and mortality in heart failure. We sought to compare LVEF and volumes assessed by BPS and RTTP, using 3D LV full volume as reference, in patients with ST-segment elevation myocardial infarction (STEMI).

Methods and results: Prospective study that included consecutive STEMI patients. LV volumes and EF were compared between BPS and RTTP and the reference method. Fifty-two patients were studied and anterior MI was the most frequent location (44\%). There was strong correlation between EF measured by BPS $(r=0.80, p<0.001)$ and RTTP $(r=0.91, p<0.001)$ versus the reference method, with significant difference between coefficients $(0.11, p=0.034)$. EF by BPS attained a good inter-operator agreement, with an intraclass correlation (ICC) of 0.82 ( $95 \% \mathrm{Cl} 0.710-0.894, p<0.001)$, as well as EF by RTTP (ICC $0.88,95 \%$ $\mathrm{Cl} 0.801-0.930, p<0.001)$, without statistically significant difference between the two methods $(p=0.279)$.

Conclusion: Estimation of LV volumes and EF by RTTP method showed higher correlation than BPS with 3D-echocardiography, in a population of STEMI patients. As an accurate LVEF estimation is of high relevance for its potential to alter clinical decision pathways, this data points to the usefulness of the RTTP method, especially in myocardial infarction setting.

\section{Introduction}

Echocardiography is a non-invasive imaging technique that provides rapid assessment of left-ventricular (LV) function. The LV function assessment through LV ejection fraction (LVEF) is of great clinical relevance in the evaluation and prognosis of patients with coronary artery disease, and plays a crucial role in the allocation of many treatments that reduce morbidity and mortality in heart failure, such as medical therapies, implantable cardioverter defibrillators or cardiac resynchronization therapy.[1-4] The biplane method of disks (Biplane Simpson, BPS) remains the currently recommended 2-dimensional (2D) method to assess LVEF and volumes.[5] However, this method has widely known limitations: 1) by using geometric assumptions about the shape of the LV and only partial information contained in two predefined tomographic planes, it makes the calculations of LV volumes and ejection fraction more inaccurate in patients in whom this information would be more clinically more relevant (geometry distorted by segmental wall motion abnormalities or aneurysms); 2) LV volume calculations by 2D BPS is highly operator dependent (both apical views' acquisition and manual tracing of endocardial border rely on the experience of the operator); [6,7] 3) it is also limited by the fact that it is not possible to acquire different projections during the same cardiac cycle. Moreover, BPS does not include the apical long-axis plane, although there are some works suggesting the benefit of a biplane evaluation including the apical 4-chamber plus apical long-axis views together.[6,8] This plane displays additional segments of the left 
ventricle (anterior septum and infero-lateral segments) which are not visualized in the apical 4 and 2chamber views. In a myocardial infarction setting where LV contractility is often unequal, this fact may be an additional limitation of the technique. The 3-dimensional (3D) real-time triplane (RTTP) potentially overcomes this issue, allowing to obtain all apical views simultaneously, at the same cardiac cycle. This method could be a more reliable and accurate alternative to BPS in LV function quantification, particularly in the scenario of myocardial infarction.

Three-dimensional based LV function measurements are recommended whenever available and feasible, especially in patients with good image quality. The technique does not use geometrical assumptions, is unaffected by foreshortening, and is more accurate and reproducible when compared to other imaging modalities. However, it has also some limitations such as lower temporal resolution, more dependency on image quality, and less published data on normal values.[5] 3D echocardiography has already shown accurate quantification of cardiac volumes and function compared with cardiac magnetic resonance imaging (MRI).[9-12] It also showed to be the most accurate technique among different ultrasound imaging modalities, for the assessment of LV function. $[9,13,14]$.

The authors sought to compare LVEF and volumes assessed by two different methods, 2Dechocardiography (BPS) and 3D-RTTP, using quantitative 3D echocardiography LV full volume as the reference method, in patients with ST-segment elevation myocardial infarction (STEMI).

\section{Materials And Methods}

\section{Study design, population and variables}

This is a prospective, cross-sectional, single-center and non-interventional study that included data from consecutive inpatients with ST segment elevation myocardial infarction (STEMI), submitted to echocardiography from March to August 2018, after stabilization and before hospital discharge. All patients underwent primary coronary intervention on the culprit vessel. Data was recorded on demographics, MI location and on echocardiography. Patients with wall motion abnormalities that corresponded to more than one coronary artery territory were classified as multivessel disease. Exclusion criteria were the presence of atrial fibrillation, significant valvular disease, left bundle branch block, ventricular pacing and poor definition of endocardial borders.

Volumes and EF measurements for left ventricle were compared between BPS and RTTP and the reference method (3D LV full volume), to assess which better correlated with it. An additional analysis of volumes and EF was performed for comparison between anterior and inferior MI location.

The study conforms to the ethical guidelines of the Declaration of Helsinki and all patients all persons gave their verbal informed consent prior to their inclusion in the study.

\section{Echocardiography}


This study was developed in a European Association of Cardiovascular Imaging (EACVI) accredited echocardiography laboratory. Echocardiographic studies were performed using Vivid E95 ultrasound equipment (GE Healthcare. General Electric Company, USA) by two experienced operators with patients in a left lateral position.

Two-dimensional echocardiography was used to calculate LVEF by Biplane Simpson. Images were acquired using a M5Sc transducer. The temporal resolution of the 2D datasets was $>50$ frames per second (fps). Triplane and 3D acquisitions were made from the apical position using the $4 \mathrm{~V}$ probe. When acquiring RTTP images, the apical 4-chamber view served as the reference image and the two other planes were by default displayed with inter-plane angles set to 60 degrees. Images were acquired during a single beat. Processing used a semi-automatic method: once the position of LV apex was identified in 4chamber apical view, it is automatically positioned in the 2-chamber and long-axis apical views for both the end-diastolic and end-systolic frames. Borders were set by manual marking of mitral valve annulus and apex in the three standard apical views. Then, three additional short-axis views were used to verify and improve the adequacy of the outline tracings, both at end-diastole and end-systole. Both operators estimated the 2D BPS and 3D RTTP for each patient blinded to clinical and angiographic data. Operators did not acknowledge each other's measurements until all post-processing was concluded. The quantitative EF evaluations of the BPS and RTTP images were made once by operator. Example available on figure $1 \mathrm{~A}$.

Three-Dimensional LV full volume datasets were acquired during four or six cardiac cycles with breathhold, depending on the spatial resolution and the cooperation of the patients. Where necessary, manual adjustments were made to the tracked endocardial border and/or to the ROI. The time markers required to identify end-diastolic and end-systolic frames were set with the help of pulsed-wave Doppler spectra of the LV outflow tract. Analyses of the 3D LV full volumes were made once by patient, using an available semi-automated analysis tool (4DLVQ, EchoPAC version 201, GE Healthcare, General Electric Company). Example available on figure 1B.

\section{Statistical analysis}

Continuous variables were expressed as mean \pm standard deviation (SD) or median (Q1-Q3), and dichotomous variables as absolute and relative frequencies. Linear regression and Pearson correlation was performed for correlation analysis. Bland-Altman plot was used for agreement assessment among the different methods. Interobserver agreement was assessed by intraclass correlation and F-test. A p value of $<0.05$ was considered statistically significant. StataCorp LP Stata/MP 14.0 was used for the statistical analysis.

\section{Results}

\section{Population characteristics}


The characteristics of the studied population are summarized in table 1. A total of 52 patients were studied, the majority of which were males in the sixth decade of life. Anterior MI was the most frequent location (44\%, 23 patients), followed by inferior MI (23\%, 12 patients).

End-systolic volume, end-diastolic volume and EF calculation were feasible in all patients. The mean EF by BPS was $44.6 \pm 10.3 \%, 47.9 \pm 16.6 \%$ by RTTP and $46.4 \pm 9.9 \%$ derived from 3D LV full volume. The median end-diastolic volume (EDV)/end-systolic volume (ESV) by BPS were $123 \mathrm{~mL} / 62 \mathrm{~mL}$, by RTTP $129 \mathrm{~mL} / 72 \mathrm{~mL}$ and by 3D full volume $120 \mathrm{~mL} / 58 \mathrm{~mL}$. These results are shown in table 2 .

\section{Biplane Simpson and Real-time Triplane comparison}

\section{Regression analysis}

There was strong correlation between EF measured by BPS and RTTP versus the reference method (3D LV full volume), with BPS reaching an $r$ coefficient of $0.80(p<0.001)$ whereas RTTP achieved an $r$ coefficient of $0.91(p<0.001)$. Linear regression data for EF is available on figure 2 . The RTTP method revealed a significantly higher $r$ coefficient than BPS (difference between $r$ coefficients $0.11, p=0.034$ ). There was also a good concordance between EDV (BPS $r=0.88, p<0.001$; RTTP $r=0.88, p<0.001$ ) and ESV (BPS $r=0.93, p<0.001$; RTTP $r=0.94, p<0.001$ ) measured by both methods, revealing similar results. No statistically significant difference between $r$ coefficients was attained.

Variability data between techniques for LVEF and volumes calculation is available on table 3 and figure 3 . For BPS and 3D-echocardiography, there was a mean difference of $2 \pm 6.1 \%$. Regarding RTTP and 3Dechocardiography, there was a mean difference of $0.6 \pm 4.2 \%$. Mean differences between methods were statistically significant $(p=0.037)$.

\section{Agreement analysis}

Detailed information on agreement analysis is available on table 4. Measurement of EF by BPS had a good inter-operator agreement, with an intraclass correlation of $0.82(95 \% \mathrm{Cl} 0.710-0.894, \mathrm{p}<0.001)$. EF by RTTP also had a good inter-operator agreement, with an intraclass correlation of $0.88(95 \% \mathrm{Cl} 0.801-$ $0.930, p<0.001)$. There was no statistically significant difference between the two methods $(p=0.279)$.

Volume quantification had good agreement for both methods. EDV calculation by BPS had an intraclass correlation of 0.90 ( $95 \% \mathrm{Cl} 0.833-0.943, \mathrm{p}<0.001)$ and by RTTP of $0.92(95 \% \mathrm{Cl} 0.855-0.951, \mathrm{p}<0.001)$. ESV calculated by BPS had an intraclass correlation $0.95(95 \% \mathrm{Cl} 0.919-0.973, p<0.001)$ and by RTTP of $0.98(95 \% \mathrm{Cl} 0.955-0.986, \mathrm{p}<0.001)$.

\section{Subsets}

RTTP had greater correlation with 3D-echocardiography than BPS in every MI subsets (table 5). However, difference between correlation coefficients was not statically significant in anterior and inferior MI 
subsets. This analysis was not made for posterolateral $\mathrm{MI}$ and patients with multivessel disease due to the smallness of the samples.

\section{Discussion}

\section{Accuracy}

The results of this study indicate that in STEMI patients with suitable echocardiographic image quality, both BPS and RTTP methods for evaluation of LVEF relate well with 3D LV full volume method. RTTP showed a significant better correlation with the reference method than BPS for the calculation of LVEF. Our study compared data from BPS and RTTP with 3D echocardiography, as the reference method. Other studies compared RTTP with cardiac MRI and achieved similar results.[15,16] For LV volumes quantification, both methods showed strong correlation with 3D full volumes. However, in our study, RTTP seemed to overestimate EDV and ESV when comparing with 3D full volume. 3D full volume seems to underestimate LV volume in comparison with cardiac MRI,[17] and the study by Malm et al.[15] showed that RTTP, unlike BPS, did not lead to LV volume underestimation, when compared with cardiac MRI. These data may contribute to explain our results. Nevertheless, these findings contrast with the results from the study by Nucifora et al.[16], which showed that both BPS and RTTP underestimated volumes in comparison with cardiac MRI.

Our results also highlights the value of RTTP for LV function evaluation in the specific scenario of myocardial infarction. In fact, this particular setting may have helped the RTTP method excel in view of BPS method because of the high prevalence of extensive wall motion abnormalities in our population. The inclusion of an additional plane (apical long-axis), which displays infero-lateral LV wall and anterior septum (the most frequently involved LV walls in patients with myocardial infarction) may help explain the greater correlation of RTTP with the 3D methodology. RTTP showed greater correlation with 3D echocardiography for EF evaluation in all MI subsets. However, the difference between $r$ coefficients was not statistically significant. It could be hypothesized if, with a greater sample, statistical significance would be attained.

\section{Reproducibility}

Some studies have shown that 3D-echocardiography has higher reproducibility than 2D-

echocardiography. $[9,17,18]$ In our study, inter-operator agreement was good for BPS and RTTP, for both EF and volumes quantification. Reliability of EF measurement by RTTP was higher than EF measured by BPS. However, difference between ICC was not significant. It may be hypothesized if with a greater sample, RTTP would attain significant difference. The better ICC of RTTP may be explained by the intrinsic characteristics of image acquisition and processing with this method, as they are more automatic and less operator dependent, which may lead to more reliability between evaluations. Our results are in consonance with similar studies. $[7,15,16]$

\section{Feasibility}


RTTP method has some intuitive advantages for EF calculation in comparison to BPS. Those are the use of the same cardiac cycle, less susceptibility to LV foreshortening, reduced acquisition time (the transducer is not moved to acquire different planes) and the acquisition of an additional view, which may increase its diagnostic accuracy (especially in myocardial infarction setting). The additional value of long-axis plane has already been addressed in some studies.[6,8] These studies have shown that using apical long-axis plane over apical 2-chamber improved feasibility, accuracy and reproducibility of LV volume and EF calculation by BPS. The information from the 3 planes altogether accomplished by RTTP may contribute to support its stronger correlation with 3D echocardiography for EF calculation.

This study contributes to the validation of RTTP's accuracy and reproducibility compared with 2Dechocardiography, even though the reference method was not MRI. RTTP is known to be inferior to 3Dechocardiography. However, RTTP also has some advantages facing 3D-echocardiography, such as not requiring a full 3D dataset over consecutive heart beats during breath-hold. This leads to faster acquisition time, less patient and operator dependency, and simpler processing (less frame-rate dependent). These characteristics are supported by good correlation with 3D-echocardiography.

\section{Study limitations}

The sample study is small but in line with similar studies. Despite that fact it was possible to achieve statistical significance in the accuracy analysis. However, neither a hypothetic better performance for EF measurement by RTTP in anterior MI subset, nor a better inter-operator agreement for RTTP could be confirmed possibly due to the small sample. The extrapolation of the data should be confirmed in largerscale studies or meta-analysis.

Our study did not include assessment of intra-operator agreement and measurement of 3D full volume was only made once by patient. However, inter-operator agreement was consistent for $2 \mathrm{D}$ and Real-time Triplane echocardiography.

This study does not have MRI data. Nevertheless, the chosen reference method (3D full volume echocardiography) has, according to published data, good correlation with MRI.[9-11]

\section{Conclusion}

Estimation of LV volumes and EF by RTTP method strongly correlate with 3D echocardiography, in a population of STEMI patients. Also, when comparing with 2D-echocardiography, RTTP method for estimation of EF showed the strongest correlation. As an accurate LVEF estimation is of utmost relevance for its potential to alter clinical decision pathways, this data points to the usefulness of the RTTP method, especially in myocardial infarction setting.

\section{Declarations}

Acknowledgements. None. 
Funding. None.

Conflict of interest. None to declare.

Availability of data and material. Available.

Authors' contributions. All of the authors contributed significantly to the study design, manuscript development and refinement, having read and approved this paper for submission. The manuscript, or part of it, has neither been published nor is currently under consideration for publication by any other journal.

Ethics approval. The study conforms to the ethical guidelines of the Declaration of Helsinki. As a noninterventional study, ethics approval is not mandatory in our institution.

Consent to participate. All patients gave their verbal informed consent prior to their inclusion in the study.

Consent for publication. All patients gave their verbal informed consent prior to their inclusion in the study.

\section{References}

1. Hammermeister KE, DeRouen TA, Dodge HT. Variables predictive of survival in patients with coronary disease. Selection by univariate and multivariate analyses from the clinical, electrocardiographic, exercise, arteriographic, and quantitative angiographic valuations. Circulation. 1979 Mar;59(3):42130. doi: 10.1161/01.cir.59.3.421. PMID: 761323.

2. Cowburn PJ, Cleland JG, Coats AJ, Komajda M. Risk stratification in chronic heart failure. Eur Heart J. 1998 May;19(5):696-710. doi: 10.1053/euhj.1997.0820. PMID: 9717002.

3. Ponikowski P, Voors AA, Anker SD, et al; ESC Scientific Document Group. 2016 ESC Guidelines for the diagnosis and treatment of acute and chronic heart failure: The Task Force for the diagnosis and treatment of acute and chronic heart failure of the European Society of Cardiology (ESC)Developed with the special contribution of the Heart Failure Association (HFA) of the ESC. Eur Heart J. $2016 \mathrm{Jul}$ 14;37(27):2129-2200. doi: 10.1093/eurheartj/ehw128. Epub 2016 May 20. Erratum in: Eur Heart J. 2016 Dec 30;: PMID: 27206819.

4. Ibanez B, James S, Agewall S, et al; ESC Scientific Document Group. 2017 ESC Guidelines for the management of acute myocardial infarction in patients presenting with ST-segment elevation: The 
Task Force for the management of acute myocardial infarction in patients presenting with STsegment elevation of the European Society of Cardiology (ESC). Eur Heart J. 2018 Jan 7;39(2):119177. doi: 10.1093/eurheartj/ehx393. PMID: 28886621.

5. Lang RM, Badano LP, Mor-Avi V, et al. Recommendations for cardiac chamber quantification by echocardiography in adults: an update from the American Society of Echocardiography and the European Association of Cardiovascular Imaging. J Am Soc Echocardiogr. 2015 Jan;28(1):1-39.e14. doi: 10.1016/j.echo.2014.10.003. PMID: 25559473.

6. Malm S, Sagberg E, Larsson H, Skjaerpe T. Choosing apical long-axis instead of two-chamber view gives more accurate biplane echocardiographic measurements of left ventricular ejection fraction: a comparison with magnetic resonance imaging. J Am Soc Echocardiogr. 2005 Oct;18(10):1044-50. doi: 10.1016/j.echo.2005.03.002. PMID: 16198881.

7. Shahgaldi K, Gudmundsson P, Manouras A, Brodin LA, Winter R. Visually estimated ejection fraction by two dimensional and triplane echocardiography is closely correlated with quantitative ejection fraction by real-time three dimensional echocardiography. Cardiovasc Ultrasound. 2009 Aug 25;7:41. doi: 10.1186/1476-7120-7-41. PMID: 19706183; PMCID: PMC2747837.

8. Nosir YF, Vletter WB, Boersma E, Frowijn R, Ten Cate FJ, Fioretti PM, Roelandt JR. The apical long-axis rather than the two-chamber view should be used in combination with the four-chamber view for accurate assessment of left ventricular volumes and function. Eur Heart J. 1997 Jul;18(7):1175-85. doi: 10.1093/oxfordjournals.eurheartj.a015414. PMID: 9243153.

9. Jenkins C, Bricknell K, Hanekom L, Marwick TH. Reproducibility and accuracy of echocardiographic measurements of left ventricular parameters using real-time three-dimensional echocardiography. J Am Coll Cardiol. 2004 Aug 18;44(4):878-86. doi: 10.1016/j.jacc.2004.05.050. PMID: 15312875.

10. Kühl HP, Schreckenberg M, Rulands D, Katoh M, Schäfer W, Schummers G, Bücker A, Hanrath P, Franke A. High-resolution transthoracic real-time three-dimensional echocardiography: quantitation of cardiac volumes and function using semi-automatic border detection and comparison with 
cardiac magnetic resonance imaging. J Am Coll Cardiol. 2004 Jun 2;43(11):2083-90. doi: 10.1016/j.jacc.2004.01.037. PMID: 15172417.

11. Mannaerts HF, Van Der Heide JA, Kamp O, Papavassiliu T, Marcus JT, Beek A, Van Rossum AC, Twisk $J$, Visser CA. Quantification of left ventricular volumes and ejection fraction using freehand transthoracic three-dimensional echocardiography: comparison with magnetic resonance imaging. $J$ Am Soc Echocardiogr. 2003 Feb;16(2):101-9. doi: 10.1067/mje.2003.7. PMID: 12574735.

12. Kitano T, Nabeshima Y, Otsuji Y, Negishi K, Takeuchi M. Accuracy of Left Ventricular Volumes and Ejection Fraction Measurements by Contemporary Three-Dimensional Echocardiography with Semiand Fully Automated Software: Systematic Review and Meta-Analysis of 1,881 Subjects. J Am Soc Echocardiogr. 2019 Sep;32(9):1105-1115.e5. doi: 10.1016/j.echo.2019.04.417. Epub 2019 Jun 21. PMID: 31230780.

13. Wood PW, Choy JB, Nanda NC, Becher H. Left ventricular ejection fraction and volumes: it depends on the imaging method. Echocardiography. 2014;31(1):87-100. doi: 10.1111/echo.12331. Epub 2013 Nov 26. PMID: 24786629; PMCID: PMC4231568.

14. Guta AC, Badano LP, Ochoa-Jimenez RC, Genovese D, Previtero M, Civera S, Ruocco A, Bettella N, Parati G, Muraru D. Three-dimensional echocardiography to assess left ventricular geometry and function. Expert Rev Cardiovasc Ther. 2019 Nov;17(11):801-815. doi: 10.1080/14779072.2019.1697234. PMID: 31770493.

15. Malm S, Frigstad S, Sagberg E, Steen PA, Skjarpe T. Real-time simultaneous triplane contrast echocardiography gives rapid, accurate, and reproducible assessment of left ventricular volumes and ejection fraction: a comparison with magnetic resonance imaging. J Am Soc Echocardiogr. 2006 Dec;19(12):1494-501. doi: 10.1016/j.echo.2006.06.021. PMID: 17138035.

16. Nucifora G, Badano LP, Dall'Armellina E, Gianfagna P, Allocca G, Fioretti PM. Fast data acquisition and analysis with real time triplane echocardiography for the assessment of left ventricular size and function: a validation study. Echocardiography. 2009 Jan;26(1):66-75. doi: 10.1111/j.15408175.2008.00762.x. Epub 2008 Nov 7. PMID: 19054037. 
17. Buccheri S, Costanzo L, Tamburino C, Monte I. Reference Values for Real Time Three-Dimensional Echocardiography-Derived Left Ventricular Volumes and Ejection Fraction: Review and Meta-Analysis of Currently Available Studies. Echocardiography. 2015 Dec;32(12):1841-50. doi: 10.1111/echo.12972. Epub 2015 Jun 6. PMID: 26053260.

18. Nikitin NP, Constantin C, Loh PH, Ghosh J, Lukaschuk El, Bennett A, Hurren S, Alamgir F, Clark AL, Cleland JG. New generation 3-dimensional echocardiography for left ventricular volumetric and functional measurements: comparison with cardiac magnetic resonance. Eur J Echocardiogr. 2006 Oct;7(5):365-72. doi: 10.1016/j.euje.2005.09.005. Epub 2006 Aug 17. PMID: 16916621.

\section{Tables}

\section{Table 1: Characteristics of the study population}

$\mathrm{HR}$, heart rate; MI, myocardial infarction

\begin{tabular}{|ll|}
\hline Variable & Total $(\mathrm{n}=52)$ \\
\hline Males, $\mathrm{n}(\%)$ & $43(82.7)$ \\
\hline Age, yo $($ mean $\pm \mathrm{sd})$ & $58.8 \pm 11.1$ \\
\hline $\mathrm{HR}, \mathrm{bpm}($ mean $\pm \mathrm{sd})$ & $63 \pm 10.7$ \\
\hline Ml location & \\
\hline Anterior, $\mathrm{n}(\%)$ & $23(44.2)$ \\
\hline Inferior, $\mathrm{n}(\%)$ & $12(23.1)$ \\
\hline Lateral, $\mathrm{n}(\%)$ & $7(13.5)$ \\
\hline Multivessel disease, $\mathrm{n}(\%)$ & $10(20.2)$ \\
\hline
\end{tabular}

\section{Table 2: LV volumes and EF measurement by the different echocardiographic methods}

LV, left ventricle; EDV, end-diastolic volume; ESV, end-systolic volume; EF, ejection fraction; BPS, Biplane Simpson; RTTP, Real-time triplane; 3D, 3-dimensional 


\begin{tabular}{|llll|}
\hline & BPS & RTTP & 3D-echocardiography \\
\hline EDV, mL (p50, IQR) & $123(106-163)$ & $129(105-168)$ & $120(89-149)$ \\
\hline$E S V, m L(p 50, I Q R)$ & $62(53-97)$ & $72(50-101)$ & $58(42-84)$ \\
\hline$E F, \%(m e a n \pm s d)$ & $44.5 \pm 9.9$ & $45.9 \pm 10$ & $46.5 \pm 9.7$ \\
\hline
\end{tabular}

Table 3: Mean differences and limit of agreement (mean \pm SD) for LV volumes and EF measurement by the different echocardiographic methods

3D, 3-dimensional; LV, left ventricle; Std, standard

\begin{tabular}{|lllll|}
\hline & Mean difference & Std deviation & Limit of agreement \\
\hline Ejection Fraction & & & & \\
\hline Biplane Simpson - 3D LV full volume & -2 & 6,1 & $-13,9$ & 9,9 \\
\hline Real-time Triplane - 3D LV full volume & $-0,6$ & 4,2 & $-8,7$ & 7,6 \\
\hline EDV & & & & \\
\hline Biplane Simpson - 3D LV full volume & 9,2 & 3,2 & $-34,3$ & 52,8 \\
\hline Real-time Triplane - 3D LV full volume & 16,3 & 2,9 & $-22,4$ & 55 \\
\hline ESV & & & & \\
\hline Biplane Simpson - 3D LV full volume & 8,2 & 2,1 & $-20,1$ & 36,4 \\
\hline Real-time Triplane - 3D LV full volume & 9,8 & 1,9 & $-15,8$ & 35,5 \\
\hline
\end{tabular}

\section{Table 4: Intraclass correlation between operators}

$E F$, ejection fraction; EDV, end-diastolic volume; ESV, end-systolic volume; ICC, intraclass correlation; $95 \%$ $\mathrm{Cl}, 95 \%$ confidence interval 


\begin{tabular}{|c|c|c|c|c|}
\hline & ICC & \multicolumn{2}{|c|}{$95 \% \mathrm{Cl}$} & $p$ value \\
\hline \multicolumn{5}{|c|}{ Biplane Simpson } \\
\hline EF & 0,822 & 0,71 & 0,894 & $<0,001$ \\
\hline EDV & 0,901 & 0,833 & 0,943 & $<0,001$ \\
\hline ESV & 0,953 & 0,919 & 0,973 & $<0,001$ \\
\hline \multicolumn{5}{|c|}{ Real-time Triplane } \\
\hline EF & 0,881 & 0,801 & 0,93 & $<0,001$ \\
\hline EDV & 0,915 & 0,855 & 0,951 & $<0,001$ \\
\hline ESV & 0,975 & 0,955 & 0,986 & $<0,001$ \\
\hline
\end{tabular}

Table 5: Correlation between EF measurement by BPS and RTTP with 3D-echocardiography in different Ml subsets

EF, ejection fraction; BPS, Biplane Simpson; RTTP, Real-time triplane; MI, myocardial infarction

\begin{tabular}{|llll|}
\hline & $r$ & Std error & $\mathrm{p}$ value \\
\hline Simpson's Biplane EF & & & \\
\hline Total & 0,80 & 0,08 & $<0,001$ \\
\hline Anterior MI & 0,78 & 0,12 & $<0,001$ \\
\hline Inferior MI & 0,74 & 0,24 & 0,006 \\
\hline Postero-lateral MI & 0,61 & 0,29 & 0,149 \\
\hline Multivessel & 0,78 & 0,13 & $<0,001$ \\
\hline Real-time Triplane & & & \\
\hline Total & 0,91 & 0,06 & $<0,001$ \\
\hline Anterior MI & 0,91 & 0,08 & $<0,001$ \\
\hline Inferior MI & 0,87 & 0,18 & $<0,001$ \\
\hline Postero-lateral Ml & 0,71 & 0,3 & 0,075 \\
\hline Multivessel & 0,92 & 0,13 & $<0,001$ \\
\hline
\end{tabular}



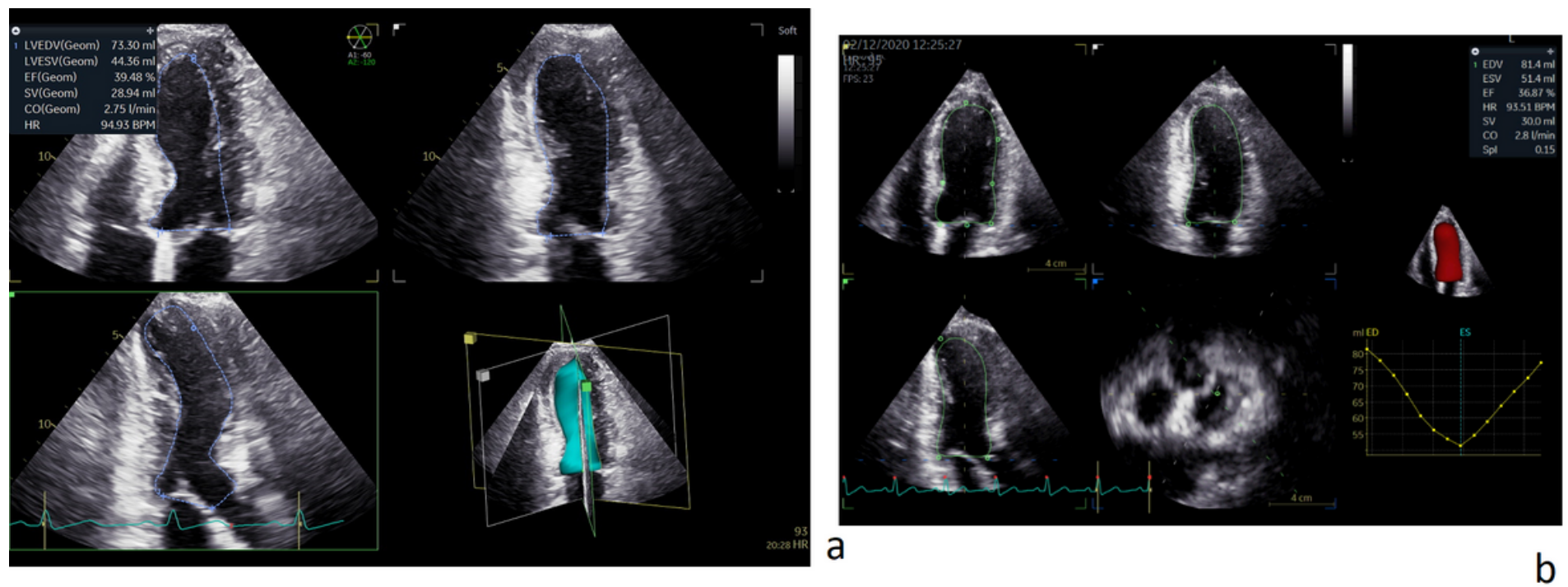

\section{Figure 1}

A. Example of Real-time Triplane ejection fraction semi-automatic determination after identification of mitral annulus and apex from 3 apical views (upper left: apical-4-chamber; upper right: apical-2-chamber; bottom left: apical-3-chamber) B. Example of left-ventricle complete 4D surface detection at end-diastole with time-volume curve using 4DLVQ.
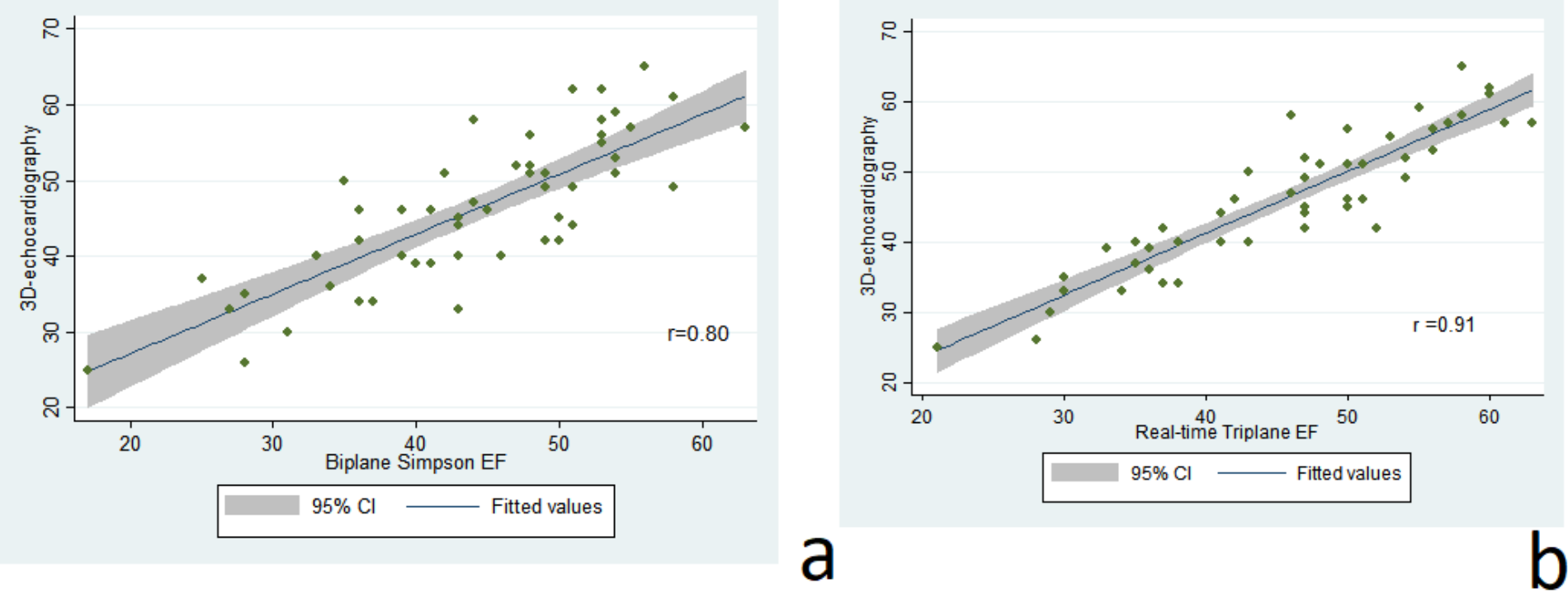

Figure 2

A: Linear regression analysis of EF measurement by Biplane Simpson and 3D-echocardiography. B: Linear regression analysis of EF measurement by Real-time Triplane and 3D-echocardiography. EF, ejection fraction 

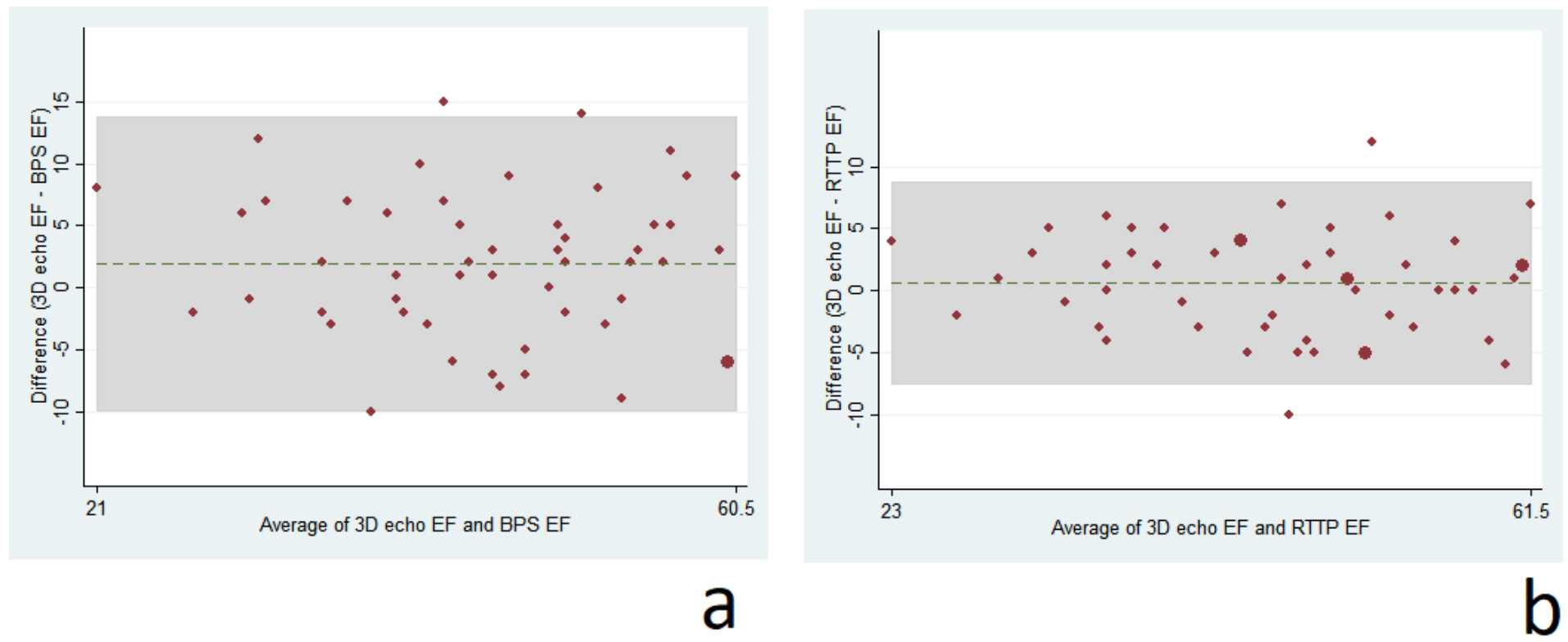

\section{Figure 3}

A: Bland-Altman plot comparing Biplane Simpson and 3D-echocardiography for the evaluation of LV EF. B: Bland-Altman plot comparing Real-time Triplane and 3D-echocardiography for the evaluation of EF. Note: y axis (difference between 3D echocardiography EF and BPS/RTTP EF) ranges from -15 to 20 in both graphs. 3D, three-dimensional; LV, left ventricle; EF, ejection fraction; BPS, Biplane Simpson; RTTP, Real-time Triplane 